\title{
Abiotic Stress-Induced Actin- Depolymerizing Factor 3 From Deschampsia antarctica Enhanced Cold Tolerance When Constitutively Expressed in Rice
}

\section{OPEN ACCESS}

Edited by:

Raul Antonio Sperotto,

Universidade do Vale do Taquari -

Univates, Brazil

Reviewed by:

Jae Sung Shim,

Chonnam National University,

South Korea

Yun Xiang,

Lanzhou University, China

*Correspondence: Woo Taek Kim

wtkim@yonsei.ac.kr

Hyoungseok Lee

soulaid@kopri.re.kr

tThese authors have contributed equally to this work

Specialty section: This article was submitted to Plant Abiotic Stress,

a section of the journal

Frontiers in Plant Science

Received: 01 July 2021

Accepted: 30 August 2021

Published: 28 September 2021

Citation:

Byun MY, Cui LH, Lee A, Oh HG, Yoo Y-H, Lee J, Kim WT and Lee H (2021)

Abiotic Stress-Induced Actin-

Depolymerizing Factor 3 From Deschampsia antarctica Enhanced Cold Tolerance When Constitutively Expressed in Rice.

Front. Plant Sci. 12:734500. doi: 10.3389/fp/s.2021.734500

\author{
Mi Young Byun ${ }^{1 \dagger}$, Li Hua Cui ${ }^{2,3+}$, Andosung Lee ${ }^{2,3}$, Hyung Geun Oh ${ }^{2,3}$, Yo-Han Yoo', \\ Jungeun Lee ${ }^{1}$, Woo Taek Kim ${ }^{2,3 *}$ and Hyoungseok Lee ${ }^{1,4 *}$ \\ ${ }^{1}$ Division of Life Sciences, Korea Polar Research Institute, Incheon, South Korea, ${ }^{2}$ Division of Life Science, Department of \\ Systems Biology, Yonsei University, Seoul, South Korea, ${ }^{3}$ Institute of Life Science and Biotechnology, Yonsei University, \\ Seoul, South Korea, ${ }^{4}$ Polar Science, University of Science and Technology, Daejeon, South Korea
}

The Antarctic flowering plant Deschampsia antarctica is highly sensitive to climate change and has shown rapid population increases during regional warming of the Antarctic Peninsula. Several studies have examined the physiological and biochemical changes related to environmental stress tolerance that allow $D$. antarctica to colonize harsh Antarctic environments; however, the molecular mechanisms of its responses to environmental changes remain poorly understood. To elucidate the survival strategies of $D$. antarctica in Antarctic environments, we investigated the functions of actin depolymerizing factor (ADF) in this species. We identified eight ADF genes in the transcriptome that were clustered into five subgroups by phylogenetic analysis. DaADF3, which belongs to a monocot-specific clade together with cold-responsive ADF in wheat, showed significant transcriptional induction in response to dehydration and cold, as well as under Antarctic field conditions. Multiple drought and low-temperature responsive elements were identified as possible binding sites of C-repeat-binding factors in the promoter region of DaADF3, indicating a close relationship between DaADF3 transcription control and abiotic stress responses. To investigate the functions of DaADF3 related to abiotic stresses in vivo, we generated transgenic rice plants overexpressing DaADF3. These transgenic plants showed greater tolerance to low-temperature stress than the wild-type in terms of survival rate, leaf chlorophyll content, and electrolyte leakage, accompanied by changes in actin filament organization in the root tips. Together, our results imply that DaADF3 played an important role in the enhancement of cold tolerance in transgenic rice plants and in the adaptation of $D$. antarctica to its extreme environment.

Keywords: abiotic stress, actin cytoskeleton, Antarctic, Deschampsia antarctica actin-depolymerizing factor 3 , low temperature, polar adaptation 


\section{INTRODUCTION}

The Antarctic monocot plant Deschampsia antarctica Desv. has a considerably wider habitat and larger numbers of populations than Colobanthus quitensis, the Antarctic dicot plant (Komárková et al., 1985). D. antarctica is highly sensitive to climate change and has shown rapid population increases and enhanced sexual reproduction success rates (Fowbert and Smith, 1994) during regional warming in the Antarctic Peninsula (Smith, 2003). Several studies have focused on the physiological and biochemical changes that allow D. antarctica to colonize harsh Antarctic environments. D. antarctica exhibits specialized morphological properties, such as distinct leaf anatomy and chloroplast ultra-structure, that influence its photosynthetic efficiency in extreme environments (Giełwanowska et al., 2005; Sáez et al., 2019), and its tolerance to ultraviolet $\mathrm{B}$-induced oxidative stress is enhanced by a combination of phenolic molecules synthesis and the activation of both enzymatic and non-enzymatic antioxidant systems (Köhler et al., 2017). The transcriptome responses of $D$. antarctica have been examined under various abiotic stress conditions (Lee et al., 2013, 2014). Increases in ice recrystallization inhibition protein (DaIRIP) expression and recrystallization inhibition activity in response to freezing stress may contribute to the cryotolerance of $D$. antarctica (John et al., 2009). In particular, the transcription factor genes DaCBF4 and DaCBF7, which regulate the expression of cold-regulated genes, can enhance plant cold tolerance when ectopically expressed in rice (Byun et al., 2015, 2018). DaGols2, which encodes a galactinol synthase that is the key enzyme mediating raffinose family oligosaccharide synthesis, improves the resistance of transgenic rice to both cold and drought (Cui et al., 2020). These findings imply that $D$. antarctica could be a model for studying the genetic and metabolic mechanisms of plant adaptations to various abiotic stresses; however, the molecular mechanisms of its adaptation to Antarctic environments remain poorly understood.

The actin cytoskeleton performs diverse cellular processes. The three-dimensional formation, disassembly, and dynamics of actin structure are regulated by diverse actin-binding proteins (Bamburg, 1999; Lappalainen, 2016). One such protein, actin-depolymerizing factor (ADF), is composed of a single folded ADF homology domain. The enzyme activity of ADF influences actin filament turnover mainly by severing actin filaments (Carlier et al., 1997). The functions of ADF/ cofilins are modulated by several factors, such as $\mathrm{pH}$ (Wioland et al., 2018), phosphorylation (Meng et al., 2004), and interaction with other actin-binding proteins (Xiang et al., 2007). Most vertebrates have one ADF and two cofilins, which are divided into muscle and non-muscle cofilins (Ono et al., 1994). Xenopus frogs express two ADF/cofilins, whereas only one ADF/cofilin gene is found in Caenorhabditis elegans (Maciver and Hussey, 2002). In contrast, plants have many more ADF/cofilin genes than animals; these are classified into multiple subgroups according to their sequence homology (Huang et al., 2020).
ADFs participate in a vast number of cellular processes, such as cell mobility (Tang and Gerlach, 2017), cytokinesis (Li et al., 2015), and plant cell growth (Hussey et al., 2006). The roles of ADF have been studied extensively in vivo among plants with large numbers of ADF genes; notably, the important roles of actin filament structures in pollen development have been analyzed in several studies (Smertenko et al., 2001; Allwood et al., 2002; Xiang et al., 2007; Jiang et al., 2019). In addition, the pollen-specific roles of AtADF7 and AtADF10 during male gametophyte development have been examined based on their spatial and temporal expression, revealing functional redundancy (Bou Daher et al., 2011). Several studies have demonstrated ADF functions in pathological responses (Inada, 2017). Actin polymerization is necessary for increasing actin filament density, which is linked to host plant susceptibility to pathogenic and non-pathogenic bacteria (Henty-Ridilla et al., 2013). The actin cytoskeleton participates in the regulation of stomatal movement, showing different patterns during stomatal opening and closing (Zhao et al., 2011). AtADF5 promotes stomatal closure through the reorganization of actin filaments in response to abscisic acid (ABA) and dehydration stress in a process mediated by the ABA signaling pathway via the ABF transcription factor DPBF3, which binds to the ADF5 promoter and turns on its gene expression (Qian et al., 2019). Vegetative profilins and ADFs are specifically upregulated by heat stress, with different expression patterns; actin filament reorganization has been observed in Arabidopsis seedlings treated with heat stress (Fan et al., 2016). Mechanical wounding has been shown to increase microfilament bundles, whereas hypoxia triggers a rapid decrease in polymerized actin and drastic inhibition of protein synthesis in potato, implying that actin cytoskeleton organization may affect translational activity under stress conditions in potato (Morelli et al., 1998).

In maize, $13 \mathrm{ZmADF}$ genes showed diverse expression patterns in various tissues in response to different stimuli, including abiotic and phytohormone stresses, implying their specific roles in plant growth, development, and external stimulus responses (Huang et al., 2020). Wheat active ADF protein was induced during cold acclimation, implying a correlation with an increased freezing tolerance in freezingtolerant wheat cultivars (Ouellet et al., 2001). OsADF3 expression was upregulated in root tissues in response to ABA or abiotic stress treatment. Transgenic Arabidopsis plants overexpressing OsADF3 showed enhanced tolerance to dehydration stress, accompanied by the upregulation of several drought tolerance response genes (Huang et al., 2012). The association of these ADF functions with exogenous stimuli, especially abiotic stresses, indicates the crucial roles of these proteins in plants under unfavorable conditions.

In this study, we investigated the functions of an $\mathrm{ADF}$ in D. antarctica, which has adapted and survived harsh Antarctic environments. Eight $A D F$ genes were identified in the transcriptome, and $D a A D F 3$ was selected based on transcriptional responses under environmental stress conditions. To investigate the functions of DaADF3 related to cold stress, we generated 
transgenic rice plants overexpressing $D a A D F 3$ and analyzed their phenotypes under low-temperature stress.

\section{MATERIALS AND METHODS}

\section{Plant Materials and Growth Conditions}

Deschampsia antarctica was collected near the King Sejong Antarctic Station $\left(62^{\circ} 14^{\prime} 29^{\prime \prime} S ; 58^{\circ} 44^{\prime} 18^{\prime \prime} \mathrm{W}\right)$ on the Barton Peninsula of King George Island in January 2007. The plants were transplanted, cultured in vitro in a tissue culture medium consisting of half-strength Murashige and Skoog (MS; Duchefa Biochemie, Haarlem, The Netherlands, 2\% sucrose, and $0.8 \%$ phytoagar; $\mathrm{pH} 5.7$ ) under a $16-\mathrm{h} / 8-\mathrm{h}$ light/dark (long-day) photoperiod with a light intensity of $150 \mu \mathrm{mol} \mathrm{m}^{-2} \mathrm{~s}^{-1}$ at $15^{\circ} \mathrm{C}$, and transferred to new medium every 3 weeks. Dry seeds from japonica rice (Oryza sativa L. cv. dongjin) were washed with $70 \%$ ethanol and then sterilized with $0.4 \% \mathrm{NaClO}$ solution. Sterilized seeds were germinated and grown on half-strength MS medium supplemented with $3 \%$ sucrose and $0.75 \%$ phytoagar $(\mathrm{pH}$ 5.7) for 10-12 days. Germinated seedlings were transplanted to soil and grown in a greenhouse at $25-30^{\circ} \mathrm{C}$ under a long-day photoperiod.

\section{Identification and Analysis of ADF Family Genes in $D$. antarctica}

Rice ADF family members were searched in the Phytozome 13 Oryza sativa v7.0 database. ${ }^{1}$ Rice ADF protein sequences were used as queries to search homologous sequences using the BLASTX program and previously reported D. antarctica transcriptome assembly data (Lee et al., 2013). Redundant sequences were discarded by manual curation. The Pfam and SMART software packages ${ }^{2}$ were used to confirm the presence of the ADF domain (SM000102). The protein isoelectric point (PI) and molecular weight $(\mathrm{Mw})$ of each gene product were calculated using the ExPasy Compute $\mathrm{pI} / \mathrm{Mw}$ tool. ${ }^{3}$ Subcellular localization of the identified proteins was analyzed using the ProtComp v9.0 program of the Softberry web tool. ${ }^{4}$ The Multiple EM for Motif Elicitation $\left(\mathrm{MEME}^{5}\right)$ online software was used to identify possible conserved motifs in the amino acid sequences of $\mathrm{DaADF}$ proteins. Putative cis-regulatory elements around 2000-bp upstream of the start codon of the $D a C B F 3$ genomic sequence (NCBI accession no. MW818101) were identified using the PlantCARE web tool. ${ }^{6}$ The NCBI BLAST search tool ${ }^{7}$ was used to find DaADF homologous sequences from other plants and to compare the identity among the eight selected

\footnotetext{
${ }^{1}$ https://phytozome-next.jgi.doe.gov/

${ }^{2} \mathrm{http}: / /$ smart.embl-heidelberg.de/

${ }^{3}$ https://web.expasy.org/compute_pi/

${ }^{4}$ http://www.softberry.com/berry.phtml?topic=protcomppl\&group=programs\&sub group $=$ proloc

${ }^{5}$ https://meme-suite.org/tools/meme

${ }^{6} \mathrm{http}: / /$ bioinformatics.psb.ugent.be/webtools/plantcare/html/

${ }^{7}$ http://www.ncbi.nlm.nih.gov/BLAST/
}

DaADF proteins. The NCBI accession numbers of the eight DaADFs are listed in Table 1.

\section{Phylogenetic Analysis}

The amino acid sequences of DaADFs and ADF homologs from monocot crops, eudicots, and bryophytes were retrieved from the GenBank database and proofread. Phylogenetic tree was constructed from the datasets using the neighbor-joining method based on the JTT matrix-based model using the MEGA X software (Kumar et al., 2018). All positions with $<95 \%$ site coverage were eliminated. We allowed $<5 \%$ alignment gaps, missing data, and ambiguous bases at any position. Support for internal branches was tested using bootstrap analyses with 1,000 replicates. The GenBank accession numbers used are listed in Supplementary Table 1.

\section{Stress Treatment}

For low-temperature treatment, $D$. antarctica plants grown at $15^{\circ} \mathrm{C}$ were transferred to a climate chamber at $4^{\circ} \mathrm{C}$ for various time periods ( $1 \mathrm{~h}$ to 7 days). For dehydration treatment, plants were transferred to filter paper, dried at $15^{\circ} \mathrm{C}$, and collected at different time points $(1,2$, and $4 \mathrm{~h})$ after the imposition of stress. For each treatment, at least two plants were used per biological replicate and a total of three biological replicates per treatment were collected. All sampling for expression analysis was conducted at the same time to avoid variation according to circadian rhythm.

\section{Real-Time Quantitative Reverse- Tanscription Polymerase Chain Reaction}

Total RNA was extracted from mature leaves of D. antarctica and rice plants using the RNeasy Plant Mini Kit (Qiagen, Hilden, Germany). The quantity and quality of RNAs were determined using an ND-1000 spectrophotometer (NanoDrop Technologies, Wilmington, DE, United States). First-strand cDNA was synthesized from $2 \mu \mathrm{g}$ total RNA using TOPscript reverse transcriptase (Enzynomics, South Korea) and oligo (dT) primers. RT-qPCR analysis was performed in $20-\mu \mathrm{l}$ reaction mixtures consisting of $1 \mu \mathrm{l} 1: 10$ diluted cDNA template, $2 \mu \mathrm{M}$ of each primer, and $10 \mu \mathrm{lTB}$ Green Premix Ex Taq (TaKaRa, Japan). The amplification procedure was as follows: denaturation and enzyme activation at $95^{\circ} \mathrm{C}$ for $5 \mathrm{~min}$, followed by 40 cycles at $95^{\circ} \mathrm{C}$ for $10 \mathrm{~s}, 55^{\circ} \mathrm{C}$ for $10 \mathrm{~s}$, and $72^{\circ} \mathrm{C}$ for $15 \mathrm{~s}$. The DaEF1a gene was used as an internal control. The DNA sequences of primers used for PCR amplification are listed in Supplementary Table 2.

\section{Subcellular Localization Assay}

The 3 ' end of the DaADF3 coding region was tagged with synthetic green fluorescent protein $(s G F P)$ in-frame and inserted into the pBI221 binary vector containing the 35S CaMV promoter. The 35S:DaADF3 construct was expressed in the $D$. antarctica protoplast using the PEG-mediated DNA transfer method. Fluorescent signals were visualized by fluorescence microscopy (BX51, Olympus, Japan). We used 35S:sGFP as a cytosolic marker. 
TABLE 1 | Detailed information of 8 actin-depolymerizing factor (ADF) family genes identified in this study.

\begin{tabular}{|c|c|c|c|c|c|c|c|c|}
\hline Gene name & Group & $\begin{array}{c}\text { NCBI } \\
\text { accession }\end{array}$ & $\begin{array}{l}\text { CDS length } \\
\text { (bp) }\end{array}$ & $\begin{array}{c}\text { Amino } \\
\text { acids (aa) }\end{array}$ & $\begin{array}{c}\text { Isoelectric } \\
\text { point }\end{array}$ & $\begin{array}{c}\text { Molecular } \\
\text { weight (kDa) }\end{array}$ & GRAVY & ProtComp \\
\hline DaADF1 & $\mathrm{C}$ & MW818093 & 420 & 139 & 5.28 & 15.94 & -0.371 & Cytoplasm and Nucleus \\
\hline DaADF2 & $\mathrm{B}$ & MW818094 & 438 & 145 & 5.43 & 16.78 & -0.467 & Cytoplasm and Nucleus \\
\hline DaADF3 & $E$ & MW818095 & 429 & 142 & 4.41 & 14.74 & -0.263 & Cytoplasm and Nucleus \\
\hline DaADF4 & $E$ & MW818096 & 429 & 142 & 5.42 & 16.14 & -0.574 & Cytoplasm and Nucleus \\
\hline DaADF5 & $A$ & MW818097 & 432 & 143 & 8.73 & 16.40 & -0.300 & Cytoplasm and Nucleus \\
\hline DaADF7 & $\mathrm{D}$ & MW818098 & 420 & 139 & 5.91 & 15.96 & -0.381 & Cytoplasm and Nucleus \\
\hline DaADF9 & $\mathrm{C}$ & MW818099 & 420 & 139 & 5.62 & 16.08 & -0.540 & Cytoplasm and Nucleus \\
\hline DaADF10 & $\mathrm{B}$ & MW818100 & 465 & 154 & 5.22 & 17.45 & -0.478 & Cytoplasm and Nucleus \\
\hline
\end{tabular}

CDS, coding sequence; bp, base pair; kDa, kilo Dalton; GRAVY, grand average of hydropathy.

\section{Generation of DaADF3-Overexpressing Transgenic Rice Plants}

Full-length coding region of DaADF3 was ligated into pGA2897 binary vector plasmids containing the maize Ubiquitin promoter (Ubi). The Ubi:DaADF3 recombinant construct was transformed into Agrobacterium tumefaciens strain LBA4404 via electroporation as previously described (Park et al., 2016). All rice transformation procedures followed established protocols as recently described by Cui et al. (2018). Generated transgenic rice T0 plants were transplanted in soil under greenhouse conditions and further propagated under paddy field conditions. The harvested transgenic seeds of individual plant were germinated in the half-strength MS medium supplemented with hygromycin B $\left(40 \mathrm{mgL}^{-1}\right)$ to select the homozygous T2 generation. Homozygous T3 DaADF3-overexpressing (independent lines $\# \mathrm{~A}, \# \mathrm{~B}$, and $\# \mathrm{C}$ ) transgenic rice progeny were used for our phenotypic analysis.

\section{Phenotype Analysis of Wild-Type (WT), Ubi:DaADF3 Rice Plants}

For low-temperature stress treatment, 5-week-old rice plants grown at $28^{\circ} \mathrm{C}$ in the same pot under a long-day photoperiod were transferred to a cold room at $4^{\circ} \mathrm{C}$. After 7 days of cold treatment, the plants were recovered at $28^{\circ} \mathrm{C}$ for $20-25$ days and their growth patterns were monitored as recently described (Cui et al., 2018). Plants that resumed growth with green, healthy leaves were regarded as having survived; the survival rates were determined at 1 month of recovery. Data were obtained from at least six biologically independent experiments. Electrolyte leakage analysis was conducted using 8-day-old whole seedlings at different cold treatment time points $(0,5$, and 10 days) at $4^{\circ} \mathrm{C}$. The cold stress-treated seedlings of WT and transgenic rice plants were soaked in a test tube containing $35 \mathrm{ml}$ distilled water on an orbital shaker $(200 \mathrm{rpm})$ at room temperature overnight. The total leaf chlorophyll (chlorophyll $\mathrm{a}+$ chlorophyll b) content of WT and transgenic rice plants was measured before and after stress treatment as described by Min et al. (2016) with slight modifications. The amounts of chlorophyll a+chlorophyll b were measured at $664.2 \mathrm{~nm}$ and $648.6 \mathrm{~nm}$, respectively, using an enzyme-linked immunosorbent assay microplate reader (VERSAmax, Molecular Devices, United States) and normalized to the dry weight of the leaves of each genotype. The electrolyte conductivity of each sample was determined following the method of Min et al. (2016). Light-grown 8-day-old whole seedlings of WT and $D a A D F 3$ overexpressors were incubated at $4^{\circ} \mathrm{C}$ for 0,5 , and 10 days. Then, the seedlings were soaked in $35 \mathrm{ml}$ distilled water at $25^{\circ} \mathrm{C}$ overnight, and electrolyte leakage was measured before and after autoclaving using a conductivity meter (Orion Star A212, Thermo Scientific, United States).

\section{Visualization of Actin Dynamics Through Fluorescence Microscopy}

Immunofluorescence experiments were conducted to detect G-actin monomers and F-actin filaments as previously described (He et al., 2006). For G-actin and F-actin labeling, root tissues were fixed by vacuum infiltration for $5 \mathrm{~min}$ in a freshly prepared solution of $1 \%$ formaldehyde in phosphate-buffered saline (PBS), kept in the fixation solution for $1 \mathrm{~h}$, and then washed in PBS three times. Then, $1-\mathrm{cm}$ pieces of the root apices were cut and incubated in a mixture of Alexa Fluor 488-DNase I (Invitrogen) and Alexa Fluor 568-phalloidin (Invitrogen) in PBS $\left(0.137 \mathrm{M} \mathrm{NaCl}, 2.7 \mathrm{mM} \mathrm{KCl}, 1.8 \mathrm{mM} \mathrm{KH} \mathrm{KO}_{4}, 10 \mathrm{mM}\right.$ $\mathrm{Na}_{2} \mathrm{HPO}_{4}, \mathrm{pH} 7.4$ ). After $10 \mathrm{~min}$, the samples were washed five times with PBS and observed using a cooled charge-coupled device camera with a laser scanning confocal microscope (LSM510 META; Carl Zeiss, Germany). The G-actin and F-actin signals were excited by lasers at 488 and $550 \mathrm{~nm}$, respectively. The fluorescence signals were measured using a sliding $0.3-\mu \mathrm{m}$ detection window in a confocal microscope.

\section{RESULTS}

\section{Identification of $A D F$ Genes in $D$. antarctica Transcriptome}

To identify the ADF gene family members in D. antarctica, we searched a previously reported transcriptome database (Lee et al., 2013) using the BLASTX program with rice ADF proteins as queries and used the SMART web tool to determine whether the ADF domain (SM000102) was present in the candidate $\mathrm{ADF}$ genes. After manual curation, eight $\mathrm{ADF}$ domain-containing genes were identified; these eight genes were designated as $D$. antarctica $A D F(D a A D F)$ and the corresponding proteins were named DaADF. A serial number was assigned to each gene according to the corresponding 
rice homolog with the highest similarity. The predicted size of DaADFs ranged from 139 to 154 amino acids, the isoelectric point ranged from 4.41 to 8.73 , and the molecular weight ranged from 14.74 to $17.45 \mathrm{kDa}$, and the grand average of hydropathy (GRAVY) values of all DaADF were $<0$, indicating that they are hydrophilic. Subcellular localization of the proteins was predicted to be multi-localized proteins, in the cytoplasm and nucleus (Table 1). The DaADFs shared $>80 \%$ sequence identity with Triticum aestivum and other monocot ADFs, ranging from 75 to $98 \%$ (Supplementary Table 3). The sequence identity among the eight DaADFs ranged from 39 to $72 \%$ (Supplementary Table 4 ).

\section{Phylogenetic Analysis of the DaADF Family}

To investigate the phylogenetic relationships among the DaADF family, we selected five flowering plants as references, including Arabidopsis thaliana, Solanum lycopersicum, Oryza sativa, Triticum aestivum, and Zea mays, as well as three bryophytes as an outgroup: Marchantia polymorpha, Physcomitrium patens, and Sphagnum fallax (Supplementary Table 1). The DaADFs were distributed in five subfamilies (Groups A to E), excluding the outgroup. DaADF5 clustered in Group A, DaADF2 and DaADF10 clustered in Group B, DaADF1 and DaADF9 clustered in Group C, DaADF7 clustered in Group D, and DaADF3 and DaADF4 clustered in Group E (Figure 1, and Table 1). The conserved motifs of DaADF family proteins were further identified using the MEME online software (Supplementary Figure 1), which predicted that DaADF family proteins contain at least four conserved motifs. Six of the eight DaADF proteins have an arrangement of motifs (order, 3-2-1-4), whereas motif 4 was missing from DaADF3 and DaADF4 in Group E, which had motif arrangements of $3-2-1-5$ and $3-2-1-7$, respectively. In summary, the overall structure of the DaADF family is highly conserved and relatively stable; however, we assume that DaADF3 and DaADF4, which belong to Group E, underwent an independent differentiation process during evolution.

\section{Expression Analysis of DaADF Genes in the Antarctic Field}

We performed gene expression analysis of the eight selected $D a A D F$ genes in D. antarctica samples previously collected in Antarctica (Cui et al., 2020) to identify genes related to adaptation to the Antarctic environment. Briefly, naturally colonized $D$. antarctica plants (field control) were collected on the Barton Peninsula of King George Island and incubated in the laboratory $\left(15^{\circ} \mathrm{C}\right.$, long-day photoperiod) for 6 days (L3d and L6d; Figure 2A). The plants were transferred back to an Antarctic field and harvested at different time points (12 h and 3 days). When the plants were transported from the field to the laboratory, the expression of DaIRIP, a cold-induced gene in D. antarctica (John et al., 2009), declined to the background level. However, the transcript levels of DaIRIP rapidly increased after the plants were transferred back to the field (from $12 \mathrm{~h}$ to 3 days; Figure 2A). Expressional changes in the eight $D a A D F$ genes under the same experimental conditions showed that the transcripts of $D a A D F 3$ were reduced in samples incubated in the laboratory, and considerably elevated in samples transferred back to the Antarctic field DaADF3 having a pattern of expressional alterations most similar to that of DaIRIP (Figure 2B).

\section{DaADF Gene Expression in Response to Cold and Drought}

Next, we investigated the expression changes of $D a A D F$ genes under low-temperature and dehydration treatment, as representative environmental stresses in Antarctica. Each stimulus was applied to $D$. antarctica plants grown at $15^{\circ} \mathrm{C}$ in the laboratory, and gene expression changes were evaluated by RT-qPCR. The genes DaADF2, DaADF3, DaADF4, DaADF5, and $D a A D F 7$ showed higher gene expression under cold stress treatment. Among these, DaADF3 showed the most significant expressional changes, reaching a peak at $8 \mathrm{~h}$ and slowly decreasing thereafter (Figure 3A). DaADF3 also displayed prominent transcriptional changes in response to drought (Figure 3B), showing a nearly 100 -fold difference compared to the control condition. In conclusion, among the eight $D a A D F$ genes identified, $D a A D F 3$ showed the largest changes in gene expression in response to cold and drought stresses. The results shown in Figures 2, 3 imply that $D a A D F 3$ encodes an $A D F$ that plays key roles in the adaptation mechanism of $D$. antarctica for survival in harsh Antarctic environmental conditions.

\section{Analysis of Cis-Acting Elements of DaADF3}

In combination with the expression characteristics of DaADF3, we analyzed cis-acting elements to explore its specificity in response to abiotic stress treatments and to provide evidence for its regulatory control at the transcriptional level. The most prominent cis-acting elements were stress-related, including multiple DRE core and ABRE elements (Supplementary Figure 2). This finding indirectly explains the high expression of $D a A D F 3$ under cold and drought stress treatment. The DaADF3 promoter region also contains light responsive elements, such as AE-box, GATA-motif, and G-box, and several hormone responsive elements, such as AuxRR-core, CGTCA-motif, and TGACG-motif, which are associated with responses to a variety of abiotic stresses.

\section{Subcellular Localization of DaADF3}

Subcellular localization of DaADF3 was investigated via a protoplast transient expression system. Protoplasts were prepared from mature leaves of $D$. antarctica plants and transfected with the 35S:DaADF3-sGFP fusion construct. The expressed proteins were visualized by fluorescence microscopy. Fluorescence signals for DaADF3 were predominantly located in the cytosolic fraction and nucleus (Figure 4), as predicted by the results shown in Table 1.

\section{Generation of DaADF3-Overexpressing Transgenic Rice Plants}

Although genetic transformation has been widely used to explore the cellular roles of stress-related genes in diverse plant species, 


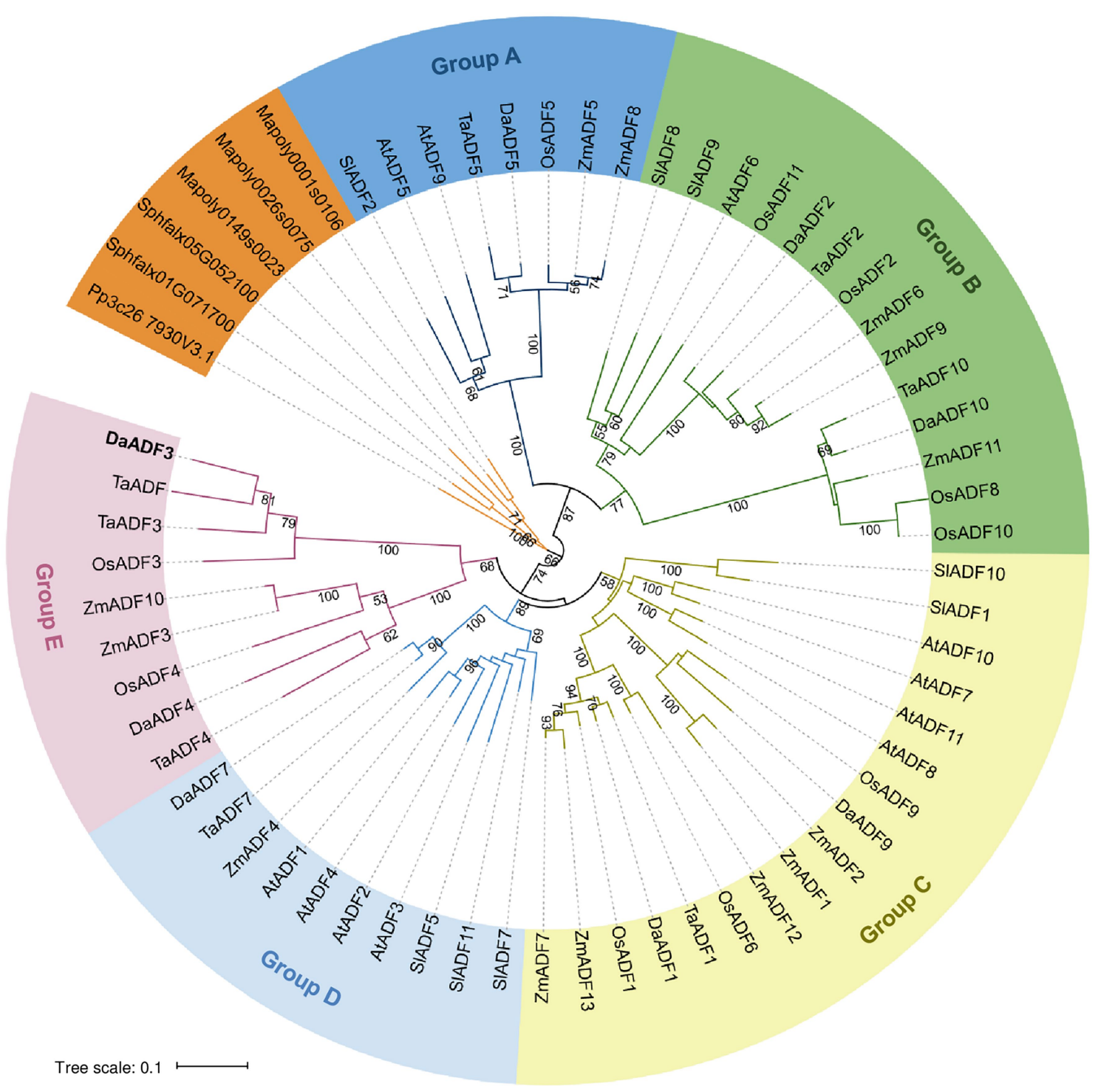

FIGURE 1 | Phylogenetic analysis of actin depolymerizing factor (ADF) proteins. The full-length amino acid sequences of DaADFs and homologs from other plants and algae were retrieved from the GenBank database and proofread. Phylogenetic trees were constructed from the datasets using the neighbor-joining method based on the JTT matrix-based model using the MEGA X software. Evolutionary distances were computed using the JTT matrix-based method. The ADF members were divided into five groups (A to $\mathbf{E}$ ).

stable gene transformation and regeneration of $D$. antarctica have not yet been established. Deschampsia antarctica and rice, a monocot model plant, belong to the same Poaceae family. Therefore, to investigate the role of DaADF3 in planta, we generated transgenic rice plants that constitutively expressed $D a A D F 3$ under the control of the maize Ubiquitin promoter (Ubi). Under normal growth conditions, the DaADF3overexpressing T3 transgenic rice plants (Ubi:DaADF3) exhibited no detectable morphological differences compared with WT rice plants. Based on the results of genomic Southern blot analysis, three independent Ubi:DaAD3F lines $\# \mathrm{~A}, \# \mathrm{~B}$, and $\# C)$ were selected, in which $D a A D F 3$ transcripts were detected by RT-PCR (Figure 5).

\section{Ubi:DaADF3 Plants Exhibited Greater Cold Stress Tolerance Than WT Plants}

To examine the cold tolerant phenotypes of the DaADF3 overexpressors, WT and T3 Ubi:DaADF3 (independent lines $\# A$, $\# B$, and $\# C$ ) rice plants were grown at $28^{\circ} \mathrm{C}$ for 5 weeks under a long-day photoperiod, transferred to a cold room at $4^{\circ} \mathrm{C}$, and incubated under continuous light. After 7 days of low-temperature treatment, these plants were transferred back to the growth room at $28^{\circ} \mathrm{C}$, allowed to recover and grow for 20-25days, while their survival was monitored. Under our experimental conditions, most of the WT rice plants exhibited discolored leaves with markedly reduced turgor after recovery from cold stress and were unable to 

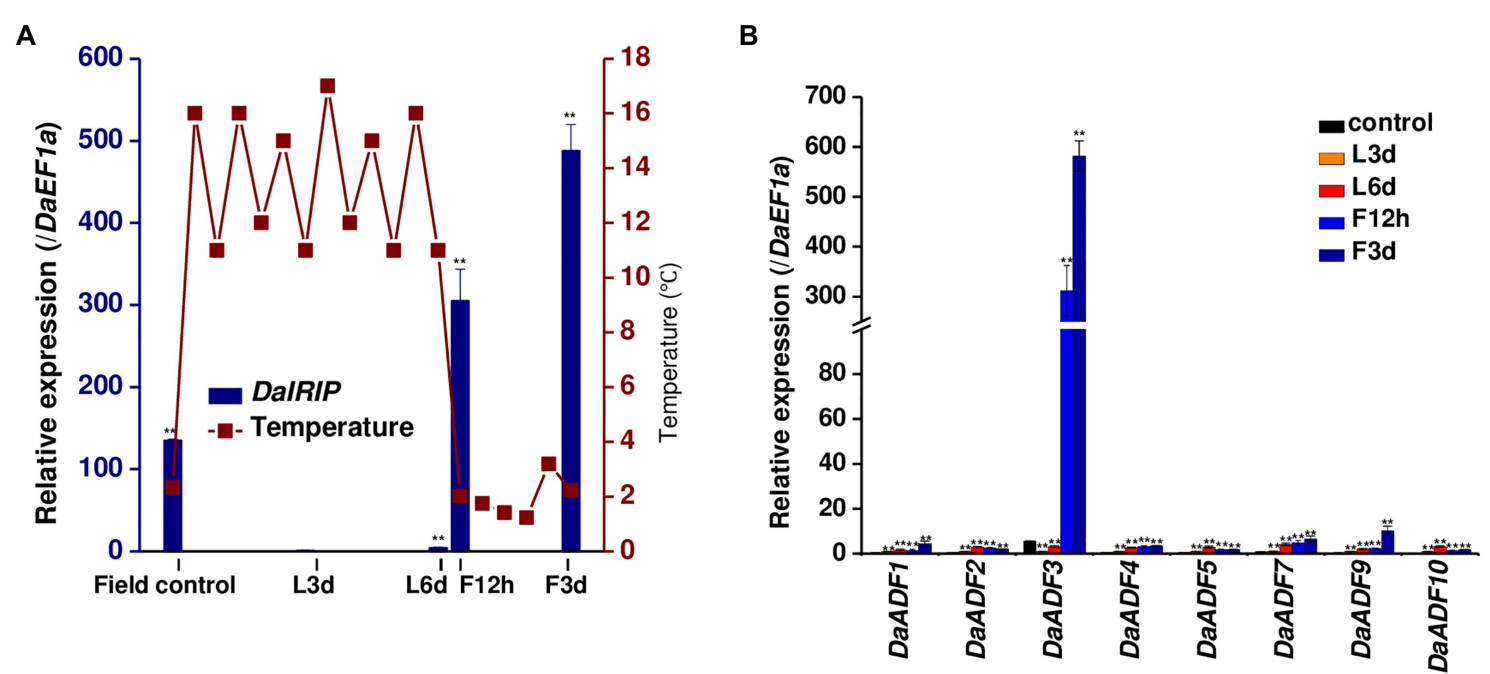

FIGURE 2 | Expression profiles of DaADF homologs in response to different growth conditions in the Antarctic field and the laboratory. (A) Expressional changes of DalRIP in response to different growth conditions. Deschampsia antarctica plants were collected in Antarctica (Field control) and incubated under laboratory conditions for 3 days (L3d) and 6 days (L6d), and then transferred back to the Antarctic field for $12 \mathrm{~h}$ (F12h) and 3 days (F3d). (B) Expression patterns of eight DaADF genes in response to the Antarctica field and laboratory conditions in $D$. antarctica plants. Total RNA was isolated from each sample and used for reversetranscription quantitative polymerase chain reaction (RT-qPCR) analysis using a gene-specific primer set (Supplementary Table 2). The relative expression of each gene was normalized to that of DaEF1a as an internal control. Data are means \pm standard deviation (SD) of three biologically independent experiments $\left({ }^{\star \star} p<0.01\right.$, Student's $t$-test).
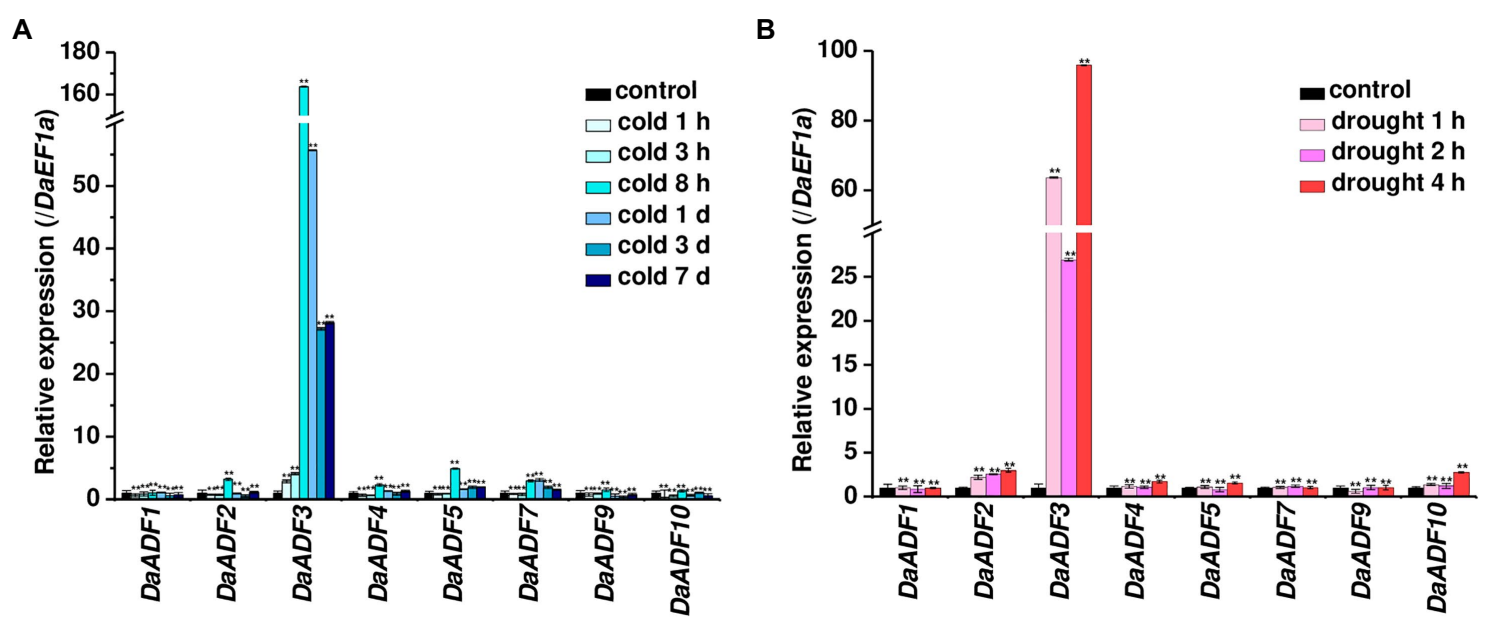

FIGURE 3 | Expression profiles of DaADF homologs in response to cold and drought. Laboratory-cultured 3-week-old D. antarctica seedlings were subjected to (A) cold $\left(4^{\circ} \mathrm{C}\right.$ for 7 days) and (B) drought (air-dried on filter paper at $15^{\circ} \mathrm{C}$ for $4 \mathrm{~h}$ ) treatments, and total RNA prepared from the treated tissues was analyzed by RTqPCR using gene-specific primer sets (Supplementary Table 2). The relative expression level of each gene was normalized to that of DaEF1a. Data are means \pm SD of three biologically independent experiments ( ${ }^{* *} p<0.01$, Student's $t$-test).

grow (survival: $8.4 \% \pm 3.0 \%$ ). In contrast, the DaADF3 overexpressors clearly showed healthier morphology and resumed growth after being relieved from cold stress (survival: $44.1 \% \pm 4.7$ to $62.3 \% \pm 6.4 \%$; Figures $\mathbf{6 A}, \mathbf{B}$ ).

To measure the total leaf chlorophyll content (chlorophyll $a+$ chlorophyll b), mature leaves were detached from plants of each genotype before and after cold treatment. Before cold treatment, the chlorophyll content of WT and
Ubi:DaADF3 plants was indistinguishable. However, the $D a A D F 3$ overexpressors contained higher amounts of chlorophyll than $\mathrm{WT}$ rice plants in response to cold temperature. After 1 month of recovery from cold treatment at $4{ }^{\circ} \mathrm{C}$, the chlorophyll content of WT leaves was $0.65 \pm 0.19 \mathrm{mg} \mathrm{g}^{-1}$ dry weight (DW), whereas the chlorophyll contents of the Ubi:DaADF3 plants ranged from $11.4 \pm 1.6$ to $11.8 \pm 0.6 \mathrm{mg} \mathrm{g}^{-1} \mathrm{DW}$ (Figure 6C). To quantify the cellular 


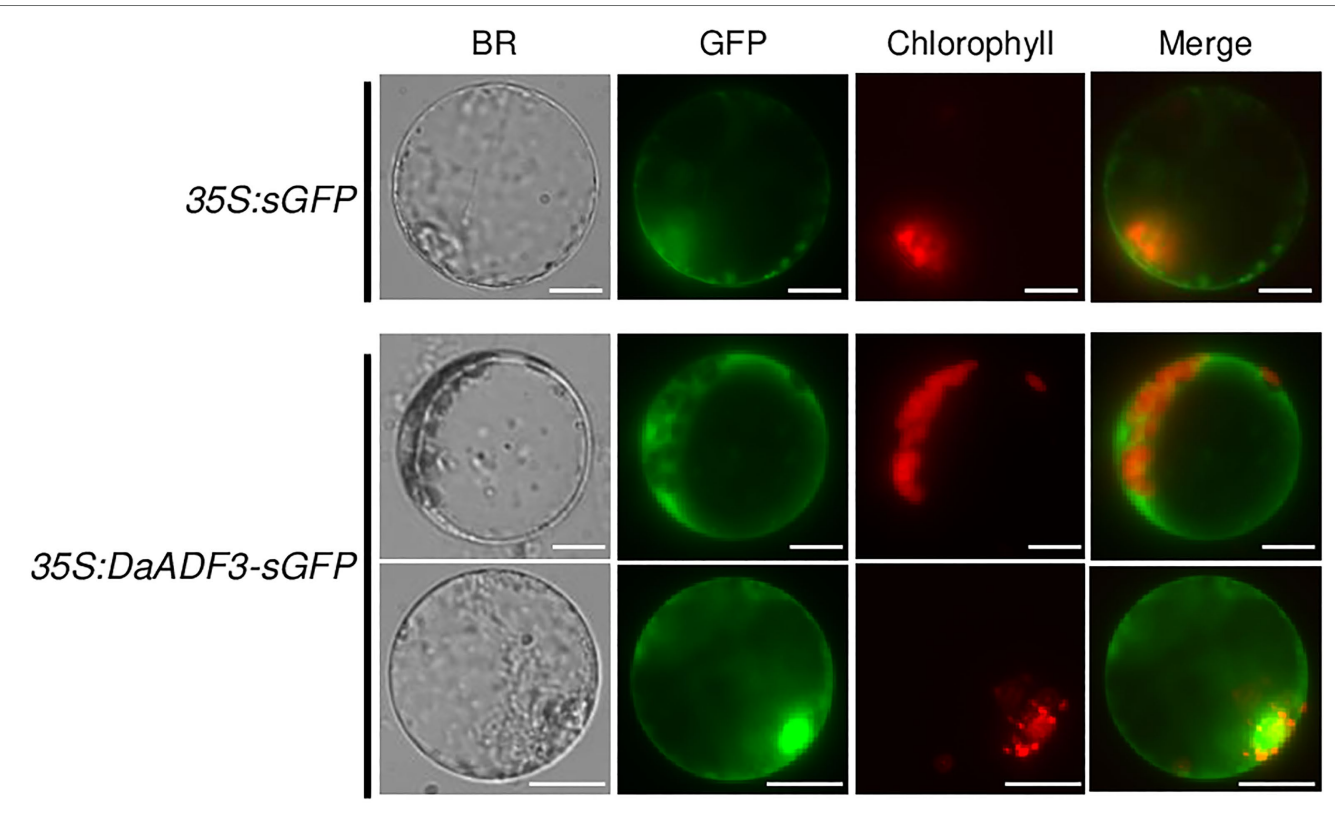

FIGURE 4 | Subcellular localization of DaADF3. The 35S:DaADF3-sGFP fusion construct was transfected into protoplasts prepared from mature leaves of $D$. antarctica. Fluorescent signals of the expressed proteins were visualized by fluorescence microscopy. $s G F P$ was $u s e d$ as a cytosolic marker protein. Bars $=20 \mu \mathrm{m}$.

response to cold stress, an electrolyte leakage assay was performed. WT seedlings showed higher ion leakage rates $(11.9 \pm 0.8 \%$ at 5 days and $21.5 \pm 2.4 \%$ at 10 days $)$ than Ubi:DaADF3 seedlings $(9.4 \pm 0.9 \%$ to $9.9 \pm 0.4 \%$ at 5 days and $9.3 \pm 1.0 \%$ to $11.4 \pm 1.2 \%$ at 10 days) in response to prolonged cold stress (Figure 6D).

To further assess the role of DaADF3 in actin cytoskeleton structural dynamics, we observed G-actin and F-actin in the root tips of WT and Ubi:DaADF3 line \#B transgenic rice plants using Alexa Fluor 488-DNase I (G-actin) and Alexa Fluor 568-phalloidin (F-actin) double labeling and laser scanning confocal microscopy. The ratio of G-actin to F-actin was found to be higher in Ubi:DaADF3 transgenic rice plants than in the WT, which indicate that DaADF3 functions in planta to depolymerize F-actin into G-actin in the root tips of transgenic plants (Figure 7).

\section{DISCUSSION}

The Wcor719 gene, which encodes ADF, has been shown to be rapidly and strongly upregulated by low temperature, with greater accumulation in tolerant winter wheat and rye cultivars than in less tolerant cultivars (Danyluk et al., 1996). Ouellet et al. (2001) detected actin depolymerization activity in the gene product and renamed Wcor 719 as TaADF. Based on the induction of an active ADF during cold acclimation and its correlation with increased freezing tolerance, the protein has been suggested to be required for cytoskeletal rearrangements occurring upon exposure to low temperatures. To determine the association between ADF and the strong cold tolerance shown by $D$. antarctica, we performed transcriptome-based screening of the ADF gene family. Unlike most animals, which have only one or two ADF/cofilins genes, plants contain a more expanded ADF gene family; $11 \mathrm{ADF}$ genes have been verified in both $A$. thaliana (Nan et al., 2017) and rice (Huang et al., 2012), and 25 genes were found in the hexaploid wheat genome (Xu et al., 2021). We isolated eight $A D F$ genes from $D$. antarctica, fewer than those found in other model plants, perhaps because we used only the transcriptome as a resource since there is presently no reference genome for this species.

ADF proteins from flowering plants have been classified into four main groups to date: rice, Arabidopsis, tomato, and wheat (Feng et al., 2006; Khatun et al., 2016; Huang et al., 2020; Xu et al., 2021). In this study, we phylogenetically classified the $D$. antarctica ADF family with model plants, such as $A$. thaliana, tomato, rice, wheat, and maize, forming five subgroups (A to E; Figure 1). In particular, group $\mathrm{E}$ was a monocotspecific clade containing TaADF and DaADF3 as the closest homologs. Similar to $T a A D F, D a A D F 3$ transcription was rapidly induced by low temperature and environmental stress in the Antarctic field (Figures 2, 3), implying that DaADF3 functions in the adaptation and colonization of $D$. antarctica in response to low temperature. This finding is consistent with a previous report that orthologous ADF gene pairs from the same subgroup have close evolutionary relationships and may preserve biological functions and transcriptional control mechanisms (Huang et al., 2020).

The direct regulation of low temperature-inducible AtADF5 by $\mathrm{CBFs}$, representative cold stress signaling transcription factors, was recently revealed in Arabidopsis. CBF proteins 
A

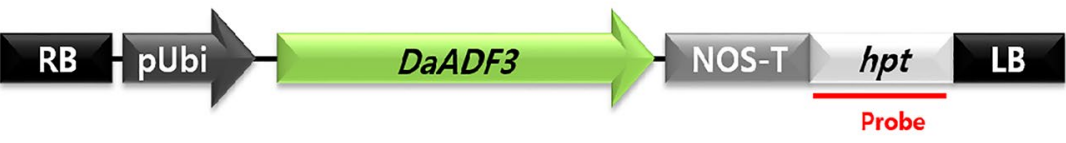

B
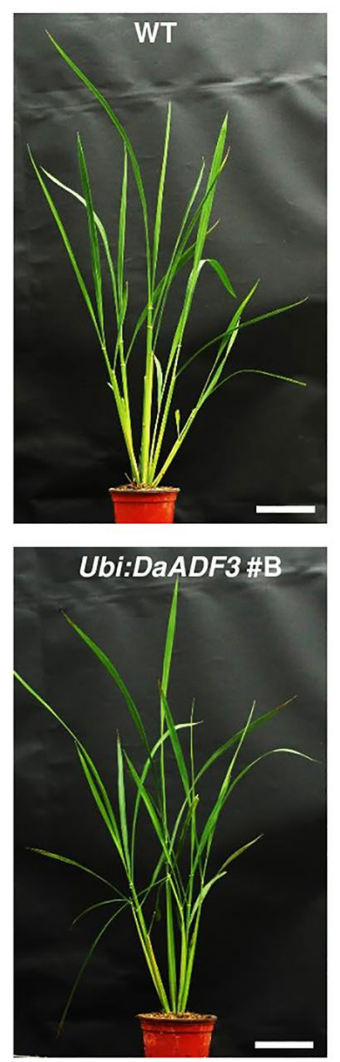
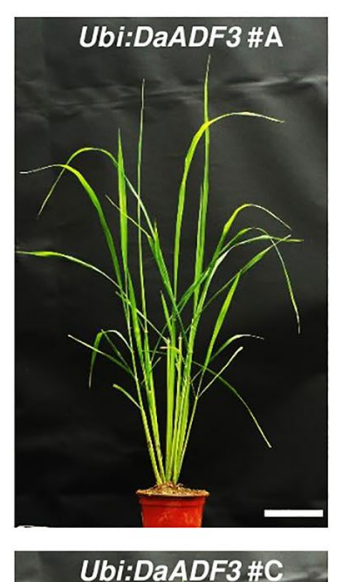

C

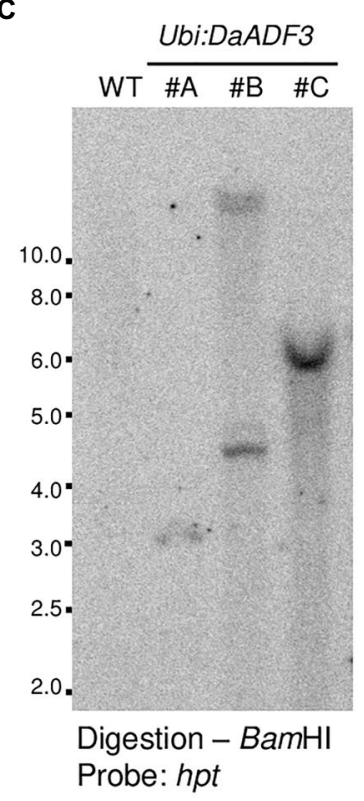

D

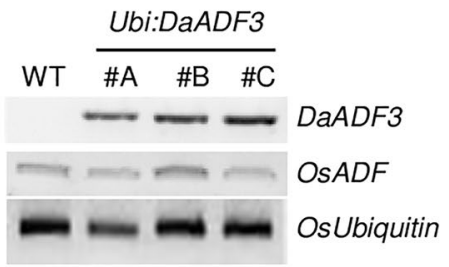

FIGURE 5 | Characterization of DaADF3-overexpressing transgenic rice plants. (A) Schematic representation of a DaADF3-overexpressing binary vector construct. Hpt, hygromycin phosphotransferase; LB, left border; pUbi, maize ubiquitin promoter; RB, right border; T-NOS, NOS terminator. (B) Morphology of 2-month-old wild-type (WT) and T3 Ubi:DaADF3 (independent lines \#A, \#B, and \#C) transgenic rice plants grown under a long-day (16-h/8-h light/dark) photoperiod.

Bars $=10 \mathrm{~cm}$. (C) Genomic Southern blot analysis. Total leaf genomic DNA was extracted from WT and T3 Ubi:DaADF3 (lines \#A, \#B, and \#C) rice plants. DNA was digested with BamHI and hybridized to a ${ }^{32} \mathrm{P}$-labeled hygromycin B phosphotransferase (hpt) probe. (D) RT-PCR analysis of the WT and T4 Ubi:DaADF3 (lines \#A, \#B, and \#C) rice plants to examine DaADF3 overexpression. OsUbiquitin was used as a loading control.

bind to the promoter and turn on the transcription of AtADF5, which is involved in regulating cytoskeleton dynamics, allowing plants to adapt to unfavorable environments (Zhang et al., 2021). DaADF3 expression increased from the beginning of cold stress treatment, peaked at $8 \mathrm{~h}$, and decreased thereafter (Figure 3A). Previous studies have reported that $D a C B F 4$ and $D a C B F 7$ transcription was immediately upregulated and soon reduced to normal states following stress treatment (Byun et al., 2015, 2018). The $D a A D F 3$ promoter contains multiple DRE and LTRE elements that are possible binding sites for CBF transcription factors (Supplementary Figure 2), and synthetic oligonucleotides designed based on the DRE element of the DaADF3 promoter have shown high binding affinity to DaCBF7 (Byun et al., 2015). Thus, we assume that DaADF3 transcription can be modulated by CBF transcription factors in the Antarctic flowering monocot plant examined in this study, and that DaADF3 further regulates actin cytoskeleton dynamics to participate in the regulation of plant adaptation to the cold Antarctic environment. However, in vivo transcriptional regulation of $D a A D F 3$ by $C B F$ transcription factors has not yet been demonstrated. Further experiments, for example, transactivation assay using DaCBF7 and DaADF3 promoter may provide more evidence for molecular regulation of $D a A D F 3$ associated to cold adaptation of D. antarctica.

DaADF3 predominantly localized to the cytosolic fraction and nucleus in protoplasts extracted from D. antarctica leaves (Figure 4). This is consistent with previous studies on subcellular localization of ADFs from wheat and cucumber (Liu et al., 2016; Tang et al., 2016; Xu et al., 2021), using the ADF protein 


\section{A}

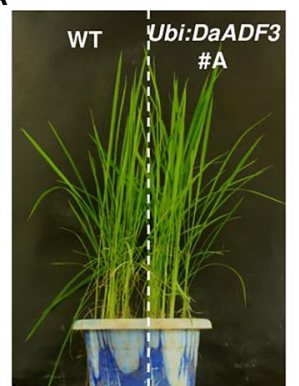

Before Cold Treatment

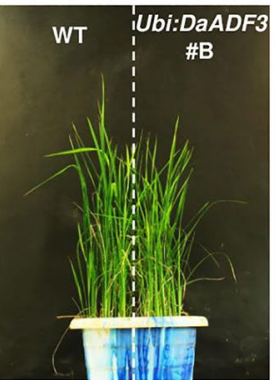

Before Cold Treatment

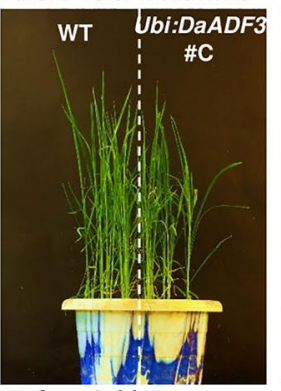

Before Cold Treatment

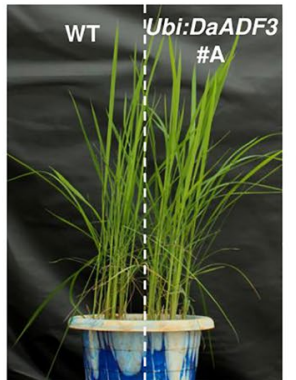

7 days cold treatment

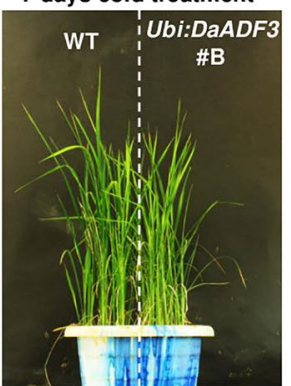

7 days cold treatment

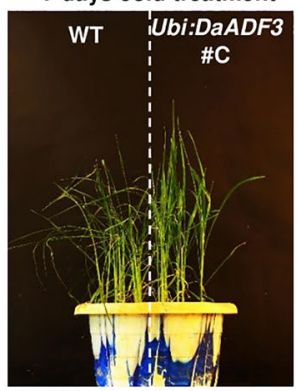

7 days cold treatment

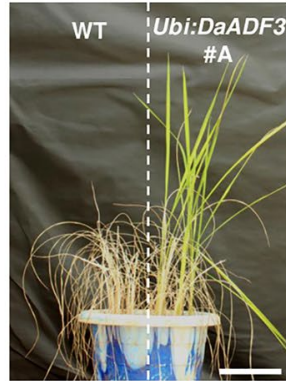

Recovery 20 days
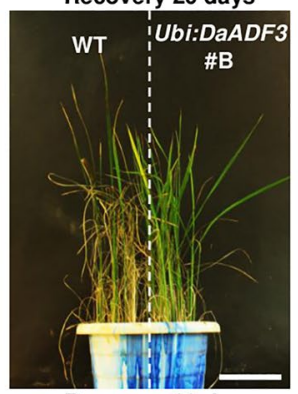

Recovery 22 days

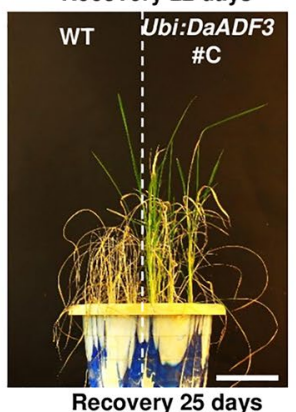

B

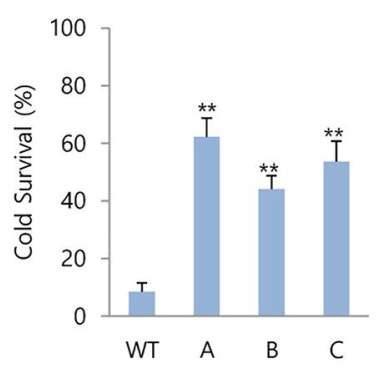

C

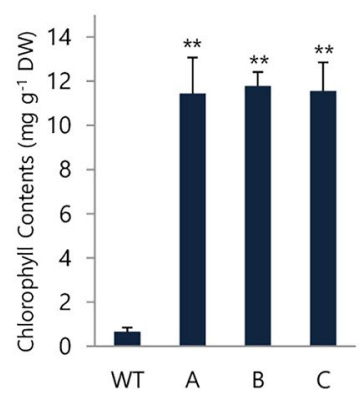

D

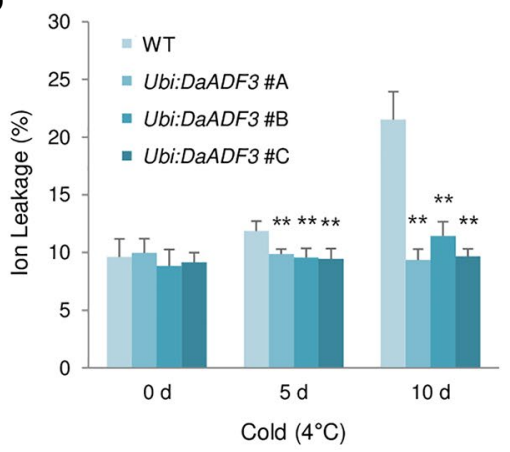

FIGURE 6 | Increased tolerance of DaADF3-overexpressing transgenic rice plants in response to cold stress. (A) Cold stress phenotypes of WT and Ubi:DaADF3 transgenic rice plants. Light-grown, 5-week-old WT and T3 Ubi:DaADF3 (lines \#A, \#B, and \#C) rice plants were transferred to a cold room at $4{ }^{\circ} \mathrm{C}$ for 8 days, after which the plants recovered at $28^{\circ} \mathrm{C}$ for $20-25$ days. Bars $=15 \mathrm{~cm}$. (B) Survival rates of WT and $U$ bi:DaADF3 plants in response to cold stress. Data are means $\pm \mathrm{SD}$ ( $n \geq 6$ biologically independent experiments; $>30$ plants per assay, ${ }^{\star *} p<0.01$, Student's $t$-test). (C) Total leaf chlorophyll content of WT and Ubi:DaADF3 plants after cold treatment. The amounts of leaf chlorophyll (chlorophyll a + chlorophyll b) were determined 1 month after recovery from cold stress. Data are means $\pm \mathrm{SD}$ ( $\mathrm{n} \geq 3$ biologically independent experiments; $>10$ plants per assay, ${ }^{* \star} p<0.01$, Student's $t$-test). (D) Electrolyte leakage analysis of WT and Ubi:DaADF3 plants before and after cold stress. Electrolyte leakage analysis was conducted using 8-day-old WT and Ubi:DaADF3 (independent lines \#A, \#B and \#C) seedlings that were incubated at $4^{\circ} \mathrm{C}$ for 0,5 , and 10 days. Data are means $\pm \mathrm{SD}(n=3$ biologically independent experiments; $>12$ plants per genotype per experiment, ${ }^{\star *} p<0.01$, Student's $t$-test).

with C-terminal GFP driven by the 35 S promoter. However, whether these GFP fusion proteins are fully functional in plant cells remains to be determined, because fusion constructs containing cofilin with GFP fused either at its $\mathrm{N}$ or $\mathrm{C}$ terminus could not complement its loss of function phenotypes in budding yeast (Okreglak and Drubin, 2007) and only intramolecular ADF-GFP fusion constructs were functional in Arabidopsis pollen tube (Zheng et al., 2013).

Ectopic expression of OsADF3 in Arabidopsis enhanced its tolerance to dehydration stress, accompanied by the upregulation of several drought tolerance response genes (Huang et al., 2012). In contrast, TaADF16 overexpression increased the freezing tolerance of transgenic Arabidopsis, possibly due to enhanced reactive oxygen species scavenging in cells. The expression levels of seven cold-response genes were found to be upregulated in transgenic Arabidopsis plants (Xu et al., 2021). In this study, transgenic rice overexpressing $D a A D F 3$ showed increased tolerance to low temperature stress compared to WT (Figure 6) and the ectopic expression of $D a A D F 3$ altered the actin cytoskeleton structure of rice plants (Figure 7). The abundance of G-actin relative to F-actin was exhibited to be higher in transgenic rice plants than in the WT, implying enhanced actin depolymerization activity in DaADF3 overexpressing plants. Furthermore, D. antarctica seedlings showed cytoskeleton structural changes in response to cold stress treatment (Supplementary Figure 3), which 


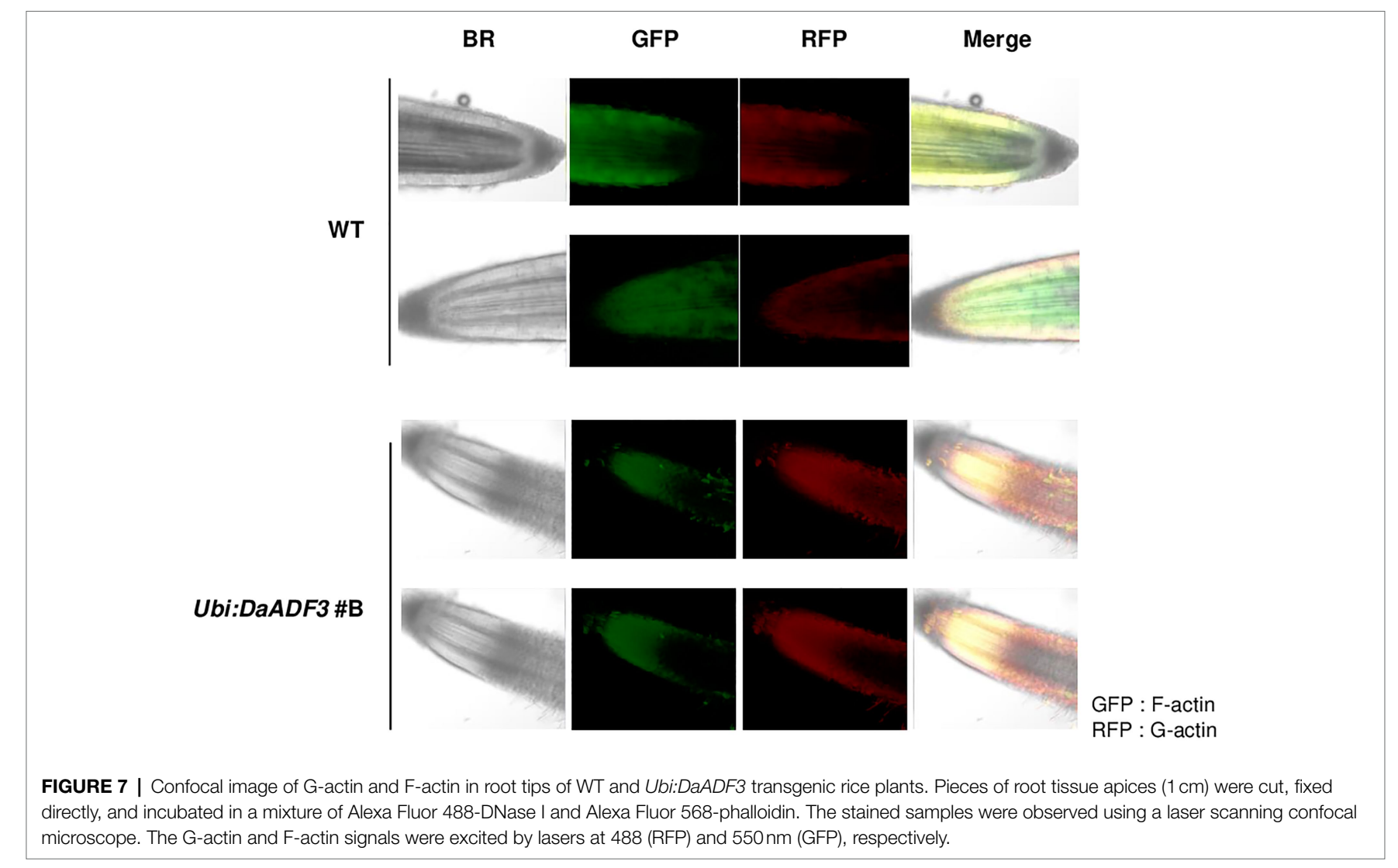

corresponds to the modulation of DaADF3 transcription under cold stress (Figure 3A). Together, these results imply that DaADF3 regulates the cytoskeleton structure to adapt to changing environmental conditions, especially cold stress in D. antarctica.

In this study, we visualized actin filament to investigate the changes of cytoskeleton in response to low temperature stimulus. As shown in Figure 7 and Supplementary Figure 3, the ratio of G-actin to F-actin was modulated in DaADF3 overexpressing rice plants and cold stress treated $D$. antarctica seedling. This might be linked to the protein activity of DaADF3, the depolymerization of actin filaments in plants. Previous studies have used chemical inhibitors of actin conformational changes, such as actin-disrupting drug latrunculin B and actin-stabilizing drug phalloidin, to elucidate their effects on actin dynamics in Arabidopsis plants in response to Pseudomonas syringae pv. tomato DC3000 (Henty-Ridilla et al., 2013) and heat stress (Fan et al., 2016). However, the effect of these inhibitors on the cold stress response of rice or D. antarctica is still unknown. Further experiments using actin-specific inhibitor may contribute to a comprehensive understanding of the action mechanism of actin cytoskeleton dynamics of $D$. antactica in response to the environmental stresses.

Cytoskeleton reorganization has been shown to be a major component of the signaling pathway under cold stress in alfalfa. The degrees of cell membrane fluidity affected the expression of the cold acclimation gene cas30, as well as calcium influx, altering its resistance to freezing stress. During cold acclimation, cytoskeleton reorganization plays a key role in cold stress responses by linking cell membrane rigidification and calcium influx to enhance survival under unfavorable conditions in Medicago sativa (Orvar et al., 2000). In a wheat thermosensitive genic male sterile (TGMS) line, male sterility was found to be strictly controlled by temperature. Cold stress repressed the transcription of cytoskeleton dynamic factors, resulting in defective cytokinesis during meiosis I. These findings may explain the induction of male sterility by low temperature in wheat TGMS line, and demonstrate the importance of cytoskeleton dynamics in the reproductive process (Tang et al., 2011). Deschampsia antarctica flourishes in harsh Antarctic environment by reproducing through autogamy, with excess pollen production (Yudakova et al., 2016). Cytoskeleton dynamics via DaADF3 may be another mechanism of action for $D$. antarctica reproduction, as it is one of only two flowering plants that have adapted to this environment.

Due to the absence of a reference genome and limited applicable genetics tools, studies on the molecular adaptation mechanisms of polar plants are far behind those of model plants. To overcome these practical limitations, we applied a combined approach, including transcriptome analysis and plant genetic transformation, and revealed the function of DaADF3 in plant cold tolerance using transgenic rice overexpressing DaADF3. Further molecular and physiological studies will be needed to determine whether CBF is the actual upstream activator that turns on $D a A D F 3$ transcription, 
and to identify the mechanism by which actin cytoskeleton changes increase plant cold tolerance.

\section{DATA AVAILABILITY STATEMENT}

The datasets presented in this study can be found in online repositories. The names of the repository/repositories and accession numbers can be found in the article/Supplementary Material.

\section{AUTHOR CONTRIBUTIONS}

WTK and HL conceived and designed the study. MYB, LHC, AL, HGO, and Y-HY performed the experiments. MYB, LHC, and JL analyzed the data. MYB, LHC, WTK, and HL discussed the results and wrote the manuscript. All authors contributed to the article and approved the submitted version.

\section{REFERENCES}

Allwood, E. G., Anthony, R. G., Smertenko, A. P., Reichelt, S., Drobak, B. K., Doonan, J. H., et al. (2002). Regulation of the pollen-specific actindepolymerizing factor LlADF1. Plant Cell 14, 2915-2927. doi: 10.1105/ tpc. 005363

Bamburg, J. R. (1999). Proteins of the ADF/cofilin family: essential regulators of actin dynamics. Annu. Rev. Cell Dev. Biol. 15, 185-230. doi: 10.1146/ annurev.cellbio.15.1.185

Bou Daher, F., van Oostende, C., and Geitmann, A. (2011). Spatial and temporal expression of actin depolymerizing factors ADF7 and ADF10 during male gametophyte development in Arabidopsis thaliana. Plant Cell Physiol. 52, 1177-1192. doi: 10.1093/pcp/pcr068

Byun, M. Y., Cui, L. H., Lee, J., Park, H., Lee, A., Kim, W. T., et al. (2018). Identification of rice genes associated with enhanced cold tolerance by comparative transcriptome analysis with two transgenic rice plants overexpressing DaCBF4 or DaCBF7, isolated from Antarctic flowering plant Deschampsia antarctica. Front. Plant Sci. 9:601. doi: 10.3389/fpls.2018.00601

Byun, M. Y., Lee, J., Cui, L. H., Kang, Y., Oh, T. K., Park, H., et al. (2015). Constitutive expression of $\mathrm{DaCBF7}$, an Antarctic vascular plant Deschampsia antarctica $\mathrm{CBF}$ homolog, resulted in improved cold tolerance in transgenic rice plants. Plant Sci. 236, 61-74. doi: 10.1016/j.plantsci.2015.03.020

Carlier, M. F., Laurent, V., Santolini, J., Melki, R., Didry, D., Xia, G. X., et al. (1997). Actin depolymerizing factor (ADF/cofilin) enhances the rate of filament turnover: implication in actin-based motility. J. Cell Biol. 136, 1307-1322. doi: $10.1083 /$ jcb.136.6.1307

Cui, L. H., Byun, M. Y., Oh, H. G., Kim, S. J., Lee, J., Park, H., et al. (2020). Poaceae type II galactinol synthase 2 from Antarctic flowering plant Deschampsia antarctica and rice improves cold and drought tolerance by accumulation of raffinose family oligosaccharides in transgenic rice plants. Plant Cell Physiol. 61, 88-104. doi: 10.1093/pcp/pcz180

Cui, L. H., Min, H. J., Byun, M. Y., Oh, H. G., and Kim, W. T. (2018). OsDIRP1, a putative RING E3 ligase, plays an opposite role in drought and cold stress responses as a negative and positive factor, respectively, in rice (Oryza sativa L.). Front. Plant Sci. 9:1797. doi: 10.3389/fpls.2018.01797

Danyluk, J., Carpentier, E., and Sarhan, F. (1996). Identification and characterization of a low temperature regulated gene encoding an actin-binding protein from wheat. FEBS Lett. 389, 324-327. doi: 10.1016/0014-5793(96)00599-6

Fan, T., Wang, R., Xiang, Y., An, L., and Cao, S. (2016). Heat stress induces actin cytoskeletal reorganization and transcript profiles of vegetative profilins and actin depolymerizing factors (ADFs) in Arabidopsis. Acta Physiol. Plant. 38:37. doi: $10.1007 / \mathrm{s} 11738-016-2061-6$

Feng, Y., Liu, Q., and Xue, Q. (2006). Comparative study of rice and Arabidopsis actin-depolymerizing factors gene families. J. Plant Physiol. 163, 69-79. doi: 10.1016/j.jplph.2005.01.015

\section{FUNDING}

This work was supported by "Post-Polar Genomics Project: Functional genomic study for securing of polar useful genes (PE21160)," funded by Korea Polar Research Institute (KOPRI), "Development of potential antibiotic compounds using polar organism resources (15250103, KOPRI Grant PM21030),” funded by the Ministry of Oceans and Fisheries, Korea, and the Basic Science Research Program, Project No. 2018R1A6A1A03025607 through the National Research Foundation (NRF), funded by the Ministry of Education, Korea.

\section{SUPPLEMENTARY MATERIAL}

The Supplementary Material for this article can be found online at: https://www.frontiersin.org/articles/10.3389/fpls.2021.734500/ full\#supplementary-material

Fowbert, J. A., and Smith, R. I. L. (1994). Rapid population increases in native vascular plants in the argentine islands, Antarctic peninsula. Arct. Alp. Res. 26, 290-296. doi: 10.2307/1551941

Giełwanowska, I., Szczuka, E., Bednara, J., and Górecki, R. (2005). Anatomical features and ultrastructure of Deschampsia antarctica (Poaceae) leaves from different growing habitats. Ann. Bot. 96, 1109-1119. doi: 10.1093/aob/mci262

He, X., Liu, Y. M., Wang, W., and Li, Y. (2006). Distribution of G-actin is related to root hair growth of wheat. Ann. Bot. 98, 49-55. doi: 10.1093/ $\mathrm{aob} / \mathrm{mcl} 084$

Henty-Ridilla, J. L., Shimono, M., Li, J., Chang, J. H., Day, B., and Staiger, C. J. (2013). The plant actin cytoskeleton responds to signals from microbeassociated molecular patterns. PLoS Pathog. 9:e1003290. doi: 10.1371/journal. ppat. 1003290

Huang, Y., Huang, W., Hong, C., Lur, H., and Chang, M. (2012). Comprehensive analysis of differentially expressed rice actin depolymerizing factor gene family and heterologous overexpression of OsADF3 confers Arabidopsis thaliana drought tolerance. Rice 5:33. doi: 10.1186/1939-8433-5-33

Huang, J., Sun, W., Ren, J., Yang, R., Fan, J., Li, Y., et al. (2020). Genome-wide identification and characterization of actin-depolymerizing factor $(A D F)$ family genes and expression analysis of responses to various stresses in Zea mays L. Int. J. Mol. Sci. 21:1751. doi: 10.3390/ijms21051751

Hussey, P. J., Ketelaar, T., and Deeks, M. J. (2006). Control of the actin cytoskeleton in plant cell growth. Annu. Rev. Plant Biol. 57, 109-125. doi: 10.1146/annurev.arplant.57.032905.105206

Inada, N. (2017). Plant actin depolymerizing factor: actin microfilament disassembly and more. J. Plant Res. 130, 227-238. doi: 10.1007/s10265-016-0899-8

Jiang, Y., Chang, M., Lan, Y., and Huang, S. (2019). Mechanism of CAP1mediated apical actin polymerization in pollen tubes. Proc. Natl. Acad. Sci. U. S. A. 116, 12084-12093. doi: 10.1073/pnas.1821639116

John, U. P., Polotnianka, R. M., Sivakumaran, K. A., Chew, O., MacKin, L., Kuiper, M. J., et al. (2009). Ice recrystallization inhibition proteins (IRIPs) and freeze tolerance in the cryophilic Antarctic hair grass Deschampsia antarctica E. Desv. Plant Cell Environ. 32, 336-348. doi: 10.1111/j.1365-3040.2009.01925.x

Khatun, K., Robin, A. H. K., Park, J. I., Kim, C. K., Lim, K. B., Kim, M. B., et al. (2016). Genome-wide identification, characterization and expression profiling of ADF family genes in Solanum lycopersicum L. Genes 7:79. doi: 10.3390/genes7100079

Köhler, H., Contreras, R. A., Pizarro, M., Cortés-Antíquera, R., and Zúñiga, G. E. (2017). Antioxidant responses induced by UVB radiation in Deschampsia antarctica Desv. Front. Plant Sci. 8:921. doi: 10.3389/fpls.2017.00921

Komárková, V., Poncet, S., and Poncet, J. (1985). Two native Antarctic vascular plants, Deschampsia antarctica and Colobanthus quitensis: a new southernmost locality and other localities in the Antarctic peninsula area. Arct. Alp. Res. 17, 401-416. doi: 10.2307/1550865 
Kumar, S., Stecher, G., Li, M., Knyaz, C., and Tamura, K. (2018). MEGA X: molecular evolutionary genetics analysis across computing platforms. Mol. Biol. Evol. 35:1547. doi: 10.1093/molbev/msy096

Lappalainen, P. (2016). Actin-binding proteins: the long road to understanding the dynamic landscape of cellular actin networks. Mol. Biol.Cell 27, 2519-2522. doi: 10.1091/mbc.e15-10-0728

Lee, J., Lee, H., Noh, E. K., Park, M., Park, H., Kim, J. H., et al. (2014). Expression analysis of transcripts responsive to osmotic stress in Deschampsia antarctica Desv. Genes Genomics 36, 283-291. doi: 10.1007/s13258-0130166-5

Lee, J., Noh, E. K., Choi, H. S., Shin, S. C., Park, H., and Lee, H. (2013). Transcriptome sequencing of the Antarctic vascular plant Deschampsia antarctica Desv. Under abiotic stress. Planta 237, 823-836. doi: 10.1007/ s00425-012-1797-5

Li, S., Sun, T., and Ren, H. (2015). The functions of the cytoskeleton and associated proteins during mitosis and cytokinesis in plant cells. Front. Plant Sci. 6:282. doi: 10.3389/fpls.2015.00282

Liu, B., Liu, X., Liu, Y., Xue, S., Cai, Y., Yang, S., et al. (2016). The infection of cucumber (Cucumis sativus L.) roots by Meloidogyne incognita alters the expression of actin-depolymerizing factor $(A D F)$ genes, particularly in association with giant cell formation. Front. Plant Sci. 7:1393. doi: 10.3389/fpls.2016.01393

Maciver, S. K., and Hussey, P. J. (2002). The ADF/cofilin family: actin-remodeling proteins. Genome Biol. 3, 1-12. doi: 10.1186/gb-2002-3-5-reviews3007

Meng, Y., Takahashi, H., Meng, J., Zhang, Y., Lu, G., Asrar, S., et al. (2004). Regulation of $\mathrm{ADF} /$ cofilin phosphorylation and synaptic function by LIMkinase. Neuropharmacology 47, 746-754. doi: 10.1016/j.neuropharm.2004. 06.030

Min, H. J., Jung, Y. J., Kang, B. G., and Kim, W. T. (2016). CaPUB1, a hot pepper u-box E3 ubiquitin ligase, confers enhanced cold stress tolerance and decreased drought stress tolerance in transgenic rice (Oryza sativa L.). Mol. Cells 39, 250-257. doi: 10.14348/molcells.2016.2290

Morelli, J. K., Zhou, W., Yu, J., Lu, C., and Vayda, M. E. (1998). Actin depolymerization affects stress-induced translational activity of potato tuber tissue. Plant Physiol. 116, 1227-1237. doi: 10.1104/pp.116.4.1227

Nan, Q., Qian, D., Niu, Y., He, Y., Tong, S., Niu, Z., et al. (2017). Plant actindepolymerizing factors possess opposing biochemical properties arising from key amino acid changes throughout evolution. Plant Cell 29, 395-408. doi: 10.1105/tpc. 16.00690

Okreglak, V., and Drubin, D. G. (2007). Cofilin recruitment and function during actin-mediated endocytosis dictated by actin nucleotide state. J. Cell Biol. 178, 1251-1264. doi: 10.1083/jcb.200703092

Ono, S., Minami, N., Abe, H., and Obinata, T. (1994). Characterization of a novel cofilin isoform that is predominantly expressed in mammalian skeletal muscle. J. Biol. Chem. 269, 15280-15286

Orvar, B. L., Sangwan, V., Omann, F., and Dhindsa, R. S. (2000). Early steps in cold sensing by plant cells: the role of actin cytoskeleton and membrane fluidity. Plant J. 23, 785-794. doi: 10.1046/j.1365-313x.2000.00845.x

Ouellet, F., Carpentier, E., Cope, M. J., Monroy, A. F., and Sarhan, F. (2001). Regulation of a wheat actin-depolymerizing factor during cold acclimation. Plant Physiol. 125, 360-368. doi: 10.1104/pp.125.1.360

Park, K. Y., Kim, E. Y., Seo, Y. S., and Kim, W. T. (2016). Constitutive expression of CaPLA1 conferred enhanced growth and grain yield in transgenic rice plants. Plant Mol. Biol. 90, 517-532. doi: 10.1007/s11103-016-0440-4

Qian, D., Zhang, Z., He, J., Zhang, P., Ou, X., Li, T., et al. (2019). Arabidopsis ADF5 promotes stomatal closure by regulating actin cytoskeleton remodeling in response to $\mathrm{ABA}$ and drought stress. J. Exp. Bot. 70, 435-446. doi: 10.1093/jxb/ery385

Sáez, P. L., Rivera, B. K., Ramírez, C. F., Vallejos, V., Cavieres, L. A., Corcuera, L. J., et al. (2019). Effects of temperature and water availability on light energy utilization in photosynthetic processes of Deschampsia antarctica. Physiol. Plant. 165, 511-523. doi: 10.1111/ppl.12739
Smertenko, A. P., Allwood, E. G., Khan, S., Jiang, C. J., Maciver, S. K., Weeds, A. G., et al. (2001). Interaction of pollen-specific actin-depolymerizing factor with actin. Plant J. 25, 203-212. doi: 10.1046/j.1365-313x.2001.00954.x

Smith, R. I. L. (2003). “The enigma of Colobanthus quitensis and Deschampsia antarctica in Antarctica," in Antarctic Biology in a Global Context. eds. A. H. L. Huiskes, W. W. C. Gieskes, J. Rozema, R. M. L. Schorno, ViesS. M. van der and W. J. Wolff (Backhuys: Leiden), 234-239.

Tang, C., Deng, L., Chang, D., Chen, S., Wang, X., and Kang, Z. (2016). TaADF3, an actin-depolymerizing factor, negatively modulates wheat resistance against Puccinia striiformis. Front. Plant Sci. 6:1214. doi: 10.3389/fpls.2015.01214

Tang, D. D., and Gerlach, B. D. (2017). The roles and regulation of the actin cytoskeleton, intermediate filaments and microtubules in smooth muscle cell migration. Respir. Res. 18, 1-12. doi: 10.1186/s12931-017-0544-7

Tang, Z., Zhang, L., Yang, D., Zhao, C., and Zheng, Y. (2011). Cold stress contributes to aberrant cytokinesis during male meiosis I in a wheat thermosensitive genic male sterile line. Plant Cell Environ. 34, 389-405. doi: $10.1111 /$ j.1365-3040.2010.02250.x

Wioland, H., Jegou, A., and Romet-Lemonne, G. (2018). Quantitative variations with $\mathrm{pH}$ of actin depolymerizing factor/Cofilin's multiple actions on actin filaments. Biochemistry 58, 40-47. doi: 10.1021/acs.biochem.8b01001

Xiang, Y., Huang, X., Wang, T., Zhang, Y., Liu, Q., Hussey, P. J., et al. (2007). ACTIN BINDING PROTEIN29 from Lilium pollen plays an important role in dynamic actin remodeling. Plant Cell 19, 1930-1946. doi: 10.1105/ tpc.106.048413

Xu, K., Zhao, Y., Zhao, S., Liu, H., Wang, W., Zhang, S., et al. (2021). Genomewide identification and low temperature responsive pattern of actin depolymerizing factor (ADF) gene family in wheat (Triticum aestivum L.). Front. Plant Sci. 12:618984. doi: 10.3389/fpls.2021.618984

Yudakova, O. I., Tyrnov, V. S., Kunakh, V. A., Kozeretskaya, I. A., and Parnikoza, I. Y. (2016). Adaptation of the seed reproduction system to conditions of maritime Antarctic in Deschampsia antarctica E. Desv. Russ. J. Dev. Biol. 47, 138-146. doi: 10.1134/S1062360416030073

Zhang, P., Qian, D., Luo, C., Niu, Y., Li, T., Li, C., et al. (2021). Arabidopsis ADF5 acts as a downstream target gene of CBFs in response to lowtemperature stress. Front. Cell Dev. Biol. 9:635533. doi: 10.3389/fcell.2021.635533

Zhao, Y., Zhao, S., Mao, T., Qu, X., Cao, W., Zhang, L., et al. (2011). The plant-specific actin binding protein SCAB1 stabilizes actin filaments and regulates stomatal movement in Arabidopsis. Plant Cell 23, 2314-2330. doi: 10.1105/tpc.111.086546

Zheng, Y., Xie, Y., Jiang, Y., Qu, X., and Huang, S. (2013). Arabidopsis ACTINDEPOLYMERIZING FACTOR7 severs actin filaments and regulates actin cable turnover to promote normal pollen tube growth. Plant Cell 25, 3405-3423. doi: $10.1105 /$ tpc.113.117820

Conflict of Interest: The authors declare that the research was conducted in the absence of any commercial or financial relationships that could be construed as a potential conflict of interest.

Publisher's Note: All claims expressed in this article are solely those of the authors and do not necessarily represent those of their affiliated organizations, or those of the publisher, the editors and the reviewers. Any product that may be evaluated in this article, or claim that may be made by its manufacturer, is not guaranteed or endorsed by the publisher.

Copyright (C) 2021 Byun, Cui, Lee, Oh, Yoo, Lee, Kim and Lee. This is an openaccess article distributed under the terms of the Creative Commons Attribution License (CC BY). The use, distribution or reproduction in other forums is permitted, provided the original author(s) and the copyright owner(s) are credited and that the original publication in this journal is cited, in accordance with accepted academic practice. No use, distribution or reproduction is permitted which does not comply with these terms. 